Voix et Images

volxetimages

\title{
Entrevue avec Jovette Marchessault
}

\section{Claudine Potvin}

Volume 16, numéro 2 (47), hiver 1991

Jovette Marchessault

URI : https://id.erudit.org/iderudit/200895ar

DOI : https://doi.org/10.7202/200895ar

Aller au sommaire du numéro

\section{Éditeur(s)}

Université du Québec à Montréal

\section{ISSN}

0318-9201 (imprimé)

1705-933X (numérique)

Découvrir la revue

\section{Citer ce document}

Potvin, C. (1991). Entrevue avec Jovette Marchessault. Voix et Images, 16(2),

218-229. https://doi.org/10.7202/200895ar d'utilisation que vous pouvez consulter en ligne.

https://apropos.erudit.org/fr/usagers/politique-dutilisation/ 


\section{Entrevue avec Jovette Marchessault}

\section{par Claudine Potvin, Université de l'Alberta}

V. \& I. - Jovette, que conservez-vous de l'enfance, des origines, et en quoi ces éléments vont-ils marquer votre écriture?

J. Marchessault - Que ma faim de consolation était impossible à rassasier. Enfant, ma vie était menacéé par plusieurs périls: d'un côté, les bouches broyeuses d'une société répressive qui haïssait les enfants et, de l'autre côté, la médiocrité. J'ai pris confiance en moi grâce surtout à ma mère et à ma grand-mère. C'est ma grand-mère qui m'a donné mes premiers livres "libres". J'entends par là les. livres que la censure romaine n'appréciait guère. Quel privilège et quelle joie que de rencontrer dans son enfance les personnages de Dickens, de Jules Verne, de Kipling, de Fenimore Cooper, de Charlotte Brontë et la petite chèvre de monsieur Séguin, et l'âne Cadichon de la comtesse de Ségur. C'est grâce à ces écrivains, écrivaines que je me suis un peu élevée au-dessus de la médiocrité. En ce qui concerne "les origines", je ne peux que répondre la première phrase de mon premier livre: Je suis d'origine céleste et je suis née à Montréal dans les années trente.

V. \& I. - À quel moment l'écriture s'est-elle imposée comme une nécessité? Que signifiait écrire pour vous à ce moment-là ? Et maintenant?

J. Marchessault - L'écriture s'est imposée comme une nécessité vers ma neuvième année et je sais que c'est aussi à cause de toutes ces lectures que le besoin de l'écriture s'est manifesté aussi tôt et aussi clairement. Je lisais comme on tombe en amour, comme on est enchanté, et à mon tour, j'ai voulu être aimée et enchanter les autres. Je voyais bien que chaque livre avait son aura unique, sa vibration presque palpable. J'ai gardé longtemps en veilleuse ce besoin d'écrire pendant que je faisais l'apprentissage de la vie. Mais je n'ai jamais renoncé à écrire et même lorsque le désespoir me disait de perdre confiance, je faisais provision d'images, de souvenirs, comme on fait des provisions en prévision de l'hiver ou de la guerre. Aujourd'hui, la faim de l'amour d'autrui s'est apaisée: je me sens moins entravée quand je pense à l'écriture. 
V. \& I. - Y a-t-il eu très tôt d'autres livres importants, à part ceux de l'enfance?

J. Marchessault - On dirait qu'à chaque année de ma vie, il y a eu des livres importants. Il y en a encore et je suis certaine qu'il y en aura jusqu'à la fin de mes jours. Il y a une telle richesse d'expression, une telle quête, dans toutes les langues, sur tous les continents. Au temps de l'adolescence, lire le Merveilleux Voyage de Nils Holgersson de Selmá Lagerlöf et quelques années plus tard, la Difficulté d'être de Jean Cocteau et Un roi sans divertissement de Jean Giono. Au commencement de lâ vingtaine, découvrir les Élégies de Duino de Rainer Maria Rilke, la Promenade au phare et la Traversée des apparences de Virginia Woolf. À trente ans, découvrir Mysterium Magnun de Jacob Boehme et quelques années plus tard l'Guvre au noir de Marguerite Yourcenar, c'est comme être amenée peu à peu à saisir une partie importante de son propre être ainsi que toute la gravité de cette vie qui peut s'élever dans des formes d'écriture, de couleurs et de sonorités qui vont vers l'indicible et l'invisible.

V. \& I. - Vous vous êtes toujours définie comme une autodidacte. Qu'implique ce processus d'autoformation pour vous?

J. Marchessault - Ce n'est pas uniquement parce que la force des circonstances et ma propre volonté m'ont portée vers ce processus d'autoformation que j'estime infiniment toutes les connaissances qui s'obtiennent par la quête, la recherche personnelle. D'une part, c'est un processus dans lequel je sens une impulsion créatrice, et d'autre part, une connaissance intuitive semble vous dire que sans l'Art, une personne vit en quelque sorte dans l'ombre d'elle-même.

V. \& I. - Vous êtes venue à l'écriture jusqu'à un certain point par la peinture et la sculpture. En quoi le travail des arts visuels et celui de l'écriture se répondent-ils? Y a-t-il un effet d'écho de l'un à l'autre?

J. Marchessault - Je crois que ce sont les arts visuels qui m'ont donné la force d'écrire. Avec la peinture et la sculpture, je me suis immédiatement sentie en contact avec un monde cosmique spirituel, psychique, cellulaire et moléculaire. Ce monde a rechargé mon âme, ma conscience, et j'ai été envahie par un sentiment de recommencement créateur.

J'avais peur de l'écriture, car c'est un art occulte, mystérieux. Comme Violette Leduc, je crois qu'écrire, c'est un peu comme faire tourner les tables. L'écriture m'incite à oser m'ouvrir à de nouvelles images même si elles sont hantées par des fantômes, des tendances inconscientes, toutes sortes d'empreintes négatives et résiduelles des siècles passés. Évidemment, si vous peignez ou si vous écrivez en laissant une soi-disant actualité l'emporter sur les potentialités ou encore en utilisant surtout vọs énergies biologiques, cette grandé expérience 
intérieure, cette espèce de mutation qui tend vers une nouvelle naissance, un nouveau commencement, n'aura pas lieu.

V. \& I. - Votre art est nourri par les éléments naturels. Que représente la campagne (les pierres, les herbes, les animaux, l'eau, le ciel, etc.) pour vous, pour votre travail?

J. Marchessault - La campagne ou si vous préférez, la Nature, nous donne des intuitions morales extraordinaires. Je ne suis pas panthéiste; pour moi, ce n'est pas chaque pierre, chaque herbe ou chaque fleur qui est d'une nature divine, mais l'énergie totalisée de toutes ces choses vivantes entre elles. C'est vraiment là que je sens qu'une intelligence divine se manifeste. J'essaie de penser et de faire et d'aimer la Nature en plantant une abondance d'arbres, de fleurs et en protégeant tout ce qui est vivant avec une vigilance qui se voudrait infatigable.

V. \& I. - Votre légitimité littéraire vous est venue d'ailleurs. En gardez-vous rancœur à la critique québécoise? Votre jugement sur les institutions scolaires, dont les universités, est assez sévère. Croyezvous à un rapport de cause à effet?

J. Marchessault - C'est possible... Cependant, je ne pense pas que la rancòeur m'aveugle quand je fais des reproches aux institutions. Mais il serait sans doute bon que je nuance davantage ma pensée. Il est très rare qu'une, ou qu'un artiste, soit reconnue par les institutions de sa collectivité quand elle œuvre en marge de l'actualité littéraire, des modes, etc. Je reproche aux institutions de succomber à ce qui brille, éclabousse et est souvent faux. Plus que tout, je reproche aux universitaires une certaine inertie. La critique est un travail sérieux: lire un livre pour en parler est pour moi un exercice autant intellectuel que spirituel. Je pense qu'on se livre à cet exercice pour soi-même d'abord et ensuite pour le bien de sa collectivité. D'une part, je pense que la critique devrait toujours dire un peu de bien du livre qu'elle n'aime pas et de l'autre, je pense qu'elle devrait prendre un peu de distance pour parler du livre qu'elle aime. C'est, il me semble, un effort nécessaire et sans cela, nous assistons au dépérissement de la critique. Au Québec, la critique tombe souvent dans la bouffonnerie: d'un côté, la critique de chapelle et de l'autre, la critique d'humeur, une humeur massacrante et méprisante. Je souhaite des échanges, des discussions et des débats.

V. \& I. - Que dire d'une certaine critique, dont le nom m'échappe, qui vous a perçue comme écrivant pour les « happy few "? Avez-vous l'impression d'être difficile à lire, compte tenu du fait que vos pièces de théâtre, et même vos romans, ne correspondent guère aux "genres traditionnels »?

J. Marchessault - Je fais beaucoup de prospection dans ce sens-là et je trouve important d'en faire. Je serai peut-être appréciée en l'an 
2000. Ce n'est pas grave. Ce qui compte pour moi, c'est de dire ce que je ressens, d'être bien dans ce que je fais, d'être bien dans ce que j'écris, dans ma vibration face aux magnifiques paysages qui m'entourent et aux influences que je reçois. J'écris avec toute l'énergie qui m'habite; voilà ce que je fais. Mais je ne peux pas dire que ça m'indiffère d'être peu lue. Cependant, il est encore plus important pour moi de ne pas avoir un rapport exploiteur avec ses lecteurs et ses lectrices, ce que font le best-seller et le théâtre d'été par exemple que je place dans la même catégorie. Au moins, les écrivains et écrivaines de la modernité n'ont pas eu ce rapport exploiteur.

V. \& I: - Comment définiriez-vous votre rapport à la langue? Ce n'est pas à proprement parler ludique, mais travaillez-vous essentiellement à partir d'un thème, d'un motif, d'une idée? Travaillez-vous autour des mots?

J. Marchessault - Non, je ne travaille pas du tout autour des mots. Je ne joue pas le «.jeu" du langage, comme on dit jouer avec le vocabulaire. Mon propos n'est pas là. J'ai des sujets et je parle de mes sujets qui, la plupart du temps, sont des femmes, du moins au théâtre. Bien sûr, il y a eu des hommes: Maurice Sachs, personnage très, très important dans La terre est trop courte, Violette Leduc, le beau personnage de Henry Miller dans Anaïs, le père dans Demande de travail sur les nébuleuses et il y a deux magnifiques personnages masculins dans Emily Carr. Donc, je m'occupe de mes sujets, de mes personnages, de mes protagonistes. Ce qui est horrible, c'est que protagoniste est un mot masculin. Ça n'existe pas au féminin; il ne peut pas y avoir de femme protagoniste dans une pièce de théatre. Je dis souvent mes protagonistes ou ma protagoniste justement pour défier ça, mais ça ne joue pas du tout avec le langage.

V. \& I. - Vous me semblez très loin de l'écriture de la modernité. Avez-vous l'impression d'y appartenir un peu même si on peut difficilement vous placer dans un groupe ou une école?

J. Marchessault - Je n'appartiens pas à la modernité. À chaque écrivaine ou écrivain ne correspond pas nécessairement une esthétique unique. L'innovation des uns et des unes est parfois moins vitale que l'approfondissement d'un arpent de terre par les autres. Je n'appartiens pas à la modernité, mais j'étais là pendant ces temps de répression et de chasse aux sorcières du sujet. Lá modernité, qui a le culte du moi, essayait de tuer l'écriture en tuant le sujet et en attirant l'attention de ses lecteurs et de ses lectrices sur tout ce qu'a d'artificiel la création littéraire. Malheureusement, tout le monde n'avait pas l'ironie et l'intelligence de Roland Barthes et il me semble que dans l'ensemble, c'était à la fois austère et ennuyeux.

V. \& I. - Diriez-vous que vous avez toujours travaillé dans la marge par rapport à l'institution littéraire québécoise? 
J. Marchessault - Géographiquement parlant, socialement parlant, sexuellement parlant, textuellement parlant, je suis dans la marge. La marge semble être le territoire des femmes en général, n'est-ce pas? Pouvons-nous être des artistes dans la solitude la plus complète? Non! Il nous faut une petite place dans le monde, au sein de la communauté humaine: cette place, c'est la place de la liberté et de la dignité. Même si nous avons contribué à déniaiser l'espace romanesque et à élargir la zone de conscience, on ne semble pas percevoir d'une façon tangible ce que nous avons fait.

V. \& I. - De fait, la création des femmes écrivaines ou autres est précisément au cœur de vos pièces de théâtre (sauf Demande de travail sur les nébuleuses) et dans Lettres de Californie. Est-ce une façon pour vous de poser le sujet féminin, l'écriture des femmes, à l'intérieur de l'histoire et de l'institution littéraire qui l'en a si souvent tenu à l'écart?

J. Marchessault - C'est ma façon de changer le monde, de participer à refaire la société en dehors des voies de la politique qui mènent presque toujours à la violence, à l'affrontement. Je crois qu'on peut changer le monde par la métaphysique et par l'art, avec des pensées, des paroles qui peuvent enthousiasmer, qui peuvent bouleverser et toucher l'humanité.

V. \& I. - Mais plus précisément, à quoi correspond l'inscription de la critique et de la théorie féministes dans vos écrits?

J. Marchessault - À un moment de répit. Avec une critique féministe qui ne souffre pas d'amnésie culturelle, j’ai pensé que des voix multiples allaient nous dire de nouveau tout ce qui se passait en littérature, en théâtre, que l'imagination était toujoưrs au pouvoir et qu'elle continuait à produire ses effets de vertige.

V. \& I. - Par rapport à ces voix, celles des femmes telluriques, des grandes déesses et de la Grand-Mère des Herbes se rejoignent-elles dans votre esprit? Renvoient-elles surtout au désir de refaire ou redire l'Histoire et à l'urgence de renouer avec une tradition et une culture ancestrales, un héritage matriarcal?

J. Marchessault - En effet, ces femmes telluriques se rejoignent toutes. Elles me font rêver à l'antique religion féministe d'Égypte et à toutes les écoles théosophistes d'Alexandrie et d'ailleurs qui contribuèrent à développer des idées de fraternité entre les peuples et à faire naître des sentiments de compassion pour les malheureux, les faibles et aussi le règne animal.

C'est aussi ma petite réponse aux Pèrès de l'Église qui sont toujours aussi phallocrates et sans imagination, sans vision puisqu'ils continuent d'identifier le principe féminin, les femmes, à leurs seins et à leur utérus, niant ainsi le grand principe maternel qui, dans toutes 
les anciennes religions, est le principe qui nourrit, qui sustente la vie de la réalité divine intérieure, en chacun, chacune de nous. Isis la grande est la maîtresse du pouvoir féminin, la Filia Vocis, la fille à la voix divine qui chante le monde dans sa manifestation. Ma réponse n'est qu'une goutte' d'eau... Et une goutte d'eau est peu de chose. Mais unie à la mer, elle ne séchera pas.

V. \& I. - L'écriture demeure donc pour vous une "mission " avant tout, la récupération de cette culture matriarcale mise à la poubelle comme vous l'écriviez ailleurs. S'agit-il toujours d'une de vos préoccupations principales?

J. Marchessault - Oui, ça reste une grande préoccupation. Mais je parlerais davantage maintenant d'une culture spirituelle. Je travaille présentement sur les textes de Madame Blavatsky, personnage de ma prochaine piècé, et je réalise que les femmes ont essayé d'instaurer en Europe à la fin du XIX siècle, grâce au culte d'Isis, la spiritualité orientale (entre autres celles de l'Inde et du bouddhisme, religions de tolérance et d'amour opposées aux religions sexistes de la tradition judéo-chrétienne).

V. \& I. - Ainsi, votre travail d'écriture consiste d'abord en grande partie à découvrir ou redécouvrir ces femmes qui ont joué un rôle dans l'histoire, comme ces aviatrices des Cailloux blancs, qui ont contribué à l'évolution de l'humanité?

J. Marchessault - Je pense que la critique de l'avenir sera karmique. On devrait pouvoir décoder tant de choses. Peut-être que dans dix ans même...

V. \& I. - Et ce serait quoi une critique karmique?

J. Marchessault - Une critique qui tienne compte des vies antérieures pour comprendre des choses. Ça suppose qu'il va falloir faire des voyages dans nos vies antérieures. J'en ai fait un il y a deux ans. Je me posais des questions sur mes textes et j'ai vu quelqu'un à ce sujet. Un pérsonnage qui n'est qu'intermédiaire, une femme très primaire qui a de la difficulté à prononcer ses mots. Je lui ai raconté que j'écrivais une pièce de théâtre sur Emily Carr et que j'en avais écrit d'autres sur un certain nombre de femmes. Je lui ai demandé comment il se faisait que j'étais si profondément rattachée à elles_et pourquoi je ressentais 'tant d'émotions à leur sujet. Elle m'a répondu que c'était arrivé il y a un peu plus de 2000 ans, au temps de Cléopâtre, non pas à sa cour, mais pas très loin d'Alexandrie. Il y avait là une assemblée de femmes qui pratiquaient une religion féministe, car la civilisation égyptienne était féministe bien que la tradition chrétienne en ait fait autre chose. Donc un groupe de femmes, vivant à part comme dans le Pique-nique sur l'Acropole de Louky Bersianik, se rassemblait régulièrement pour discuter de questions féministes. Elles essayaient 
de trouver, par la réflexion et la méditation, des solutions aux problèmes de l'humanité, mais dans un esprit de joie et de célébration; dans le rire. Une petite fille orpheline se trouvait parmi elles et elles l'adoptèrent. C'était moi et elles m'avaient tant donné que je leur ai demandé comment je pourrais le leur rendre. Comme elles croyaient toutes à la réincarnation, elles m'ont dit: "Un jour, tu écriras et tu parleras de nous."

V. \& I. - Vous récupérez également dans vos récits un savoir ancien, souvent de source amérindienne, qui consiste à nommer la terre, la faune, la flore, les mythes, etc. Tout est-il donc perdu ou constamment à redire comme on se réapproprie nos territoires?

J. Marchessault - Je crois que dans la plupart des cosmologies, le processus de création est exécuté au moyen du son, de la parole, du mot. En Inde, Vishnu est appelé "la Voix ", car c'est le grand chanteur qui construit le monde et l'univers par son chant. On raconte que quand la voix cosmique parle, les mondes se font et que tant qu'elle parlera, les mondes se feront. Nous mêlons nos voix narratives, j'en suis persuadée, à ce chant cosmique. La longue phrase de la Création n'est pas terminée, d'où l'apparente imperfection présente. J'imagine qu'un jour, quand la grande phrase sera terminée, nous aurons le plus parfait des systèmes solaires et que nous pourrons tous et toutes changer de règne et passer à un plan moins dense.

V. \& I. - À quels choix ou désirs correspondent l'écriture narrative ou la forme dramatique? Qu'est-ce qui vous fait privilégier le récit ou le théâtre?

J. Marchessault - Je n'ai pas l'impression de faire consciemment un choix. Quelqu'un parle près de moi, en moi. Est-ce la voix d'une ombre, d'une étoile, d'un esprit, je ne sais. Autour de moi, à la campagne, tout semble baigner dans l'art, tout semble pouvoir se transformer en formes artistiques. Au commencement, il y a cette impulsion vers une forme ou une autre. Théâtre ou roman? Je pense que le roman est bien plus sorcier, plus mystérieux, plus envoûtant... mais le théâtre est plus humain, plus tragique, d'un tragique intérieur aussi profond que celui du roman sans doute, mais sur lequel vous avez moins de contrôle. Contrairement au roman, le théâtre se passe au niveau du sol, mais soudain, grâce au talent combiné de plusieurs âmes humaines, le texte se transforme en une vie vibrante de couleurs et de formes. La_création du monde, quelle grande et belle pièce de théâtre!

V. \& I. - En ce sens, croyez-vous que votre trilogie romanesque tend à présenter une vision utopique de la destinée humaine (triomphe de la lumière et de l'amour sur la noirceur et le mal, réconciliation véritable entre femmes et hommes, etc., pour reprendre les termes de Gloria Orenstein à propos du troisième roman)? 
J. Marchessault - L'avenir de l'humanité est une chose dont le développement et la splendeur n'ont pas de limites. Pour envisager cette possibilité, encore faut-il être capable d'échapper à l'inertie de nos institutions, à la peur. Si nous laissons tout cela nous étouffer, nous ne pourrons jamais innover d'une façon consciente.

V. \& I. - Cependant, la trilogie s'articule autour d'un récit de nature autobiographique. À quelle nécessité répond ce discours du "je" longuement raconté ici?

J. Marchessault - Le «je " de la trilogie n'est pas celui de Job angoissé par ses péchés et espérant malgré tout le salut. Il n'est pas le composant socio-biologique que l'on comprend habituellement en "je", " moi ", et il n'est pas non plus identifiable avec les fonctions du corps ou enfermé dans des formes sociales et culturelles. D'une façon peutêtre plus évidente dans Des cailloux blancs pour les forêts obscures, le «je" tente de s'imprégner d'une nouvelle conception des relations humaines et des liens qui nous unissent au monde animal, végétal et minéral. C'est un monde qui n'existe pas encore, mais à force non seulement de le penser, mais surtout de le parler, de l'écrire, ce «je" qui est aussi celui de l'humanité, le fera exister.

V. \& I. - Le motif du voyage traverse ces textes, et les pièces de théâtre également d'ailleurs. Considérez-vous vos textes narratifs comme le récit d'une traversée, voire d'un voyage initiatique au cours duquel la narratrice/auteure se trouve "initiée" aux secrets de la création et retrouve ainsi la mémoire du temps?

,J. Marchessault - Les livres que je trouve les plus beaux, les plus émouvants, font le récit de cette traversée «des apparences" et tant d'écrivaines et d'écrivains m'ont imprégnée, influencée, inspirée. Par exemple, tout le Journal d'Anaïs Nin, cette parfaite "métis", cette femme cosmopolite, est le récit de ce Voyage qui la mènera jusqu'au bord de la mer de la côte Ouest américaine où elle va s'éteindre en méditant sur le mystère des rencontres, des relations humaines.

V. \& I. - Tout cela est très présent dans les longs périples de nombreux personnages des Cailloux blancs. Comment rattachez-vous la quête du couple lesbien au motif de l'ange et à la figure du père, je devrais dire de Dieu le père?

J.-Marchessault --En fait, c'est pour ça que le rapport aux hommes est tellement difficile. Parce qu'il s'agit bien de Dieu le père mais en réalité, le règne du père n'est pas arrivé. C'est le règne de l'esprit. Je le dis souvent, en tant que filles, quand on parle de nos pères, quand on pense à eux, ils s'avèrent souvent bien décevants en fonction de ce qu'on attend d'eux. Mais les hommes aussi attendent le règne de la mère qui nourrit la vie intérieure.

V. \& I. - Dans ce cas, ce serait l'ange annonciateur? 
J. Marchessault - Oui, mais l'ange de la présénce, l'ange gardien, l'esprit-guide, la lumière du monde. Le Lion de Bangor, c'est une surprise pour moi. Je croyais faire quinze pages avec lui; il restait là et chaque jour, je voyageais plus loin avec lui. Il prenait substance, il devenait chair, tellement vrai qu'il serait venu frapper à ma porte que ça ne m'aurait pas étonnée. Il fait à lui seul les trois quarts du livre et il est le seul à pouvoir répondre aux questions de Jeanne. C'est vraiment lui la rencontre avec l'ange, la rencontre avec l'esprit; il représente l'esprit.

V. \& I. - Serait-ce que le monde lesbien ou l'écriture lesbienne telle que présentée dans Tryptique lesbien (sic) ne correspond plus à votre champ d'exploration et qu'elle disparaît au profit d'une relation autre?

J. Marchessault - Je n'en suis plus là. Je me sens comme une âme qui écrit sur d'autres âmes. Toute connotation sexuelle s'efface. D'autre part, je n'ai plus envie d'enfourcher ce cheval de bataille.

V. \& I. - Pourquoi l'ange de la présence se manifeste-t-il à travers la mort?

J. Marchessault - Parce que la mort, c'est l'áu-delà et que c'est très près pour moi. L'au-delà, c'est très présent; les femmes parlent à travers lui (la mère de Noria, Harriet).

V. \& I. - Ce père, ou ce que vous en faites, me semble tout de même un personnage bien ambigu, équivoque. Faudrait-il le rapprocher du père dans Demande de travail sur les nébuleuses, jugé trop fin, trop aimable, selon les féministes, et trop radical par les autres?

J. Marchessault - C'est le point où j'en suis rendue dans mon évolution. J'ai envie de faire des personnages nuancés. Il n'y a que les hommes pour dire certaines choses. Dans Demande de travail sur les nébuleuses, la mère ne pouvait pas dire ce que le père disait parce que ça ne lui appartient pas, ce n'est pas son univers. Lui parle d'autre chose. Ce que je trouvais essentiel de montrer, c'est l'évolution entre trois générations d'hommes, le grand-père, le père, le fils, le dernier étant chômeur spirituel, diplômé universitaire refusant de transmettre son savoir et cherchant sa place sur la terre. Ça m'étonne qu'on conçoive le texte comme un discours féministe radical d'une part et une concession dé l'autre. Les gens ont peut-être des préjugés vis-à-vis de moi, des attentes, comme si on attendait quelque chose de moi et qu'on n'écoutait pas ce que je dis.

V. \& I. - Cette pièce marque une sorte de virement dans votre production. La concevez-vous comme une pièce sage, un texte de la maturité?

J. Marchessault - Demande de travail sur les nébuleuses est sur la même note vibratoire que mon roman Des cailloux blancs pour les forêts obscures qui l'a', en quelque sorte, enfantée. C'est 
une prospection du futur: au milieu de rien mais aussi beau, aussi froid et clair qu'une dernière neige, ce vingt et unième siècle qui les hante déjà! La mère, qui a une longueur d'avance sur les autres, écrit des lettres d'amour aux étoiles. Sa fille est allée demander son identité aux vieilles pierres des Andes alors que son frère, barman à New York, se dit chômeur spirituel. Le père, ouvrier de la onzième heure, est celui qui peut le mieux pressentir combien l'agressivité de l'ego, le succès matériel et la soif humaine de confort et de pouvoir, combien tous ces désirs pulsionnels ont pollué l'éther et intoxiqué les esprits. C'est sans doute une pièce de la maturité où j'essaie de concilier le "naturalisme " ou "réalisme " et le merveilleux.

V. \& I. - Pourriez-vous nous dire quelques mots de plus au sujet de votre dernière pièce sur Emily Carr?

J. Marchessault - Le Voyage magnifique d'Emily Carr est celui de cette peintre de la côte Ouest qui habitait un lieu magique qu'elle avait baptisé la Maison de toutes les espèces. C'est là qu'Emily accueillait les visiteurs de la planète: sa sœur aînée Lizzie, avec des rebuffades et des cris de guerre, devient l'adversaire au même titre que l'étouffante société victorienne; son amie indienne Sophie, espritguide qui apporte les messages, mais aussi les devoirs et les leçons de l'existence; son jeune ami peintre du Groupe des Sept, Lawren Harris, qui veut débarrasser la peinture de ses dogmes et révolutionner l'art au Canada; et celui qui vibre à son appel et répond à ses pensées d'adoration et de compassion.

Il me semble être dans la continuité de cette enfant de la Terre qui, au commencement d'une trilogie, voyageait dans le ventre d'un lévrier alors qu'Emily voyagera, elle, dans le ventre d'un éléphant qui représente la stabilité et l'immuabilité. Dans la culture orientale, ces valeurs sont précisément celles qui bénéficient de la plus grande considération, contrairement à ce qui se passe chez nous où l'on privilégie le dynamisme et le changement. La thématique du "voyage" est la plus belle métaphore de la Vie, celle qui polarise les plus fortes énergies

V. \& I. - On/je cherche le corps dans vos textes et on ne le trouve pas toujours. Il semble pris, inscrit, figé même, dans la terre ou dans les gestes scandés de vos personnages de théâtre. Est-ce que je me trompe?

J. Marchessault - Parfois, il est un peu plus présent comme dans la fête sacrée des vaches de nuit, ou encore dans la Saga des poules mouillées, mais en ce qui me concerne, le corps est une terrible limitation. Une malédiction en quelque sorte! Tout le dernier chant de Comme une enfant de la terre raconte l'angoisse de passer d'un monde familier - celui des étoiles - dans un univers inconnu. Quitter la plénitude sans bornes du ciel pour les limitations terrestres. 
Comment apaiser cette angoisse, comment s'en consoler? En ouvrant la narration au flot de vie qui nous soulève tout en restant fidèle au plan mystérieux qui nous habite? Peut-être...

V. \& I. - Finalement, le corps, serait-ce davantage pour vous le pays, l'étendue, le large, les pistes géographiques du cœur humain, l'Amérique, l'Arctique, espaces complémentaires plutôt qu'oppositionnels?

J. Marchessault - En Inde, on raconte cette merveilleuse fable: un jour, Shiva, pour plaisanter, essaya de faire s'affronter ses deux fils dans une compétition. Il les défia de faire le tour du monde et de voir lequel était le plus rapide. Au signal du départ, Skanda son fils s'élança comme une flèche tandis que Ganesh, son autre fils, se mit à se promener dans le plus grand calme en tournant autour de sa mère Parvati et de son père. Tourner autour de ses parents, dit Ganesh, équivaut à faire le tour du monde, si bien que lorsque Skanda revint, haletant et exténué, de son tour du monde, Ganesh avait déjà été proclamé vainqueur. On ne pouvait en attendre moins de Ganesh, ce dieu à tête d'éléphant qui est le dieu de la connaissance.

V. \& I. - Quels livres circulent le plus sur votre table de travail ces jours-ci?

J. Marchessault - Comme je l'ai mentionné auparavant, j'écris une pièce sur Héléna Petrovna Blavatsky, dont le titre est Madame Blavatsky, spirite. Je suis donc plongée dans les écrits de mon héroïne qui sont nombreux et admirables d'intelligence, d'inspiration et de connaissances. Je découvre en même temps toute l'effervescence spirituelle du XIX ${ }^{\mathbf{e}}$ siècle, le choc des idées, la lutte des femmes pour le contrôle des naissances et l'égalité des sexes. Madame Blavatsky, qui est russe, a dominé à mon avis le mouvement intellectuel et spirituel de son siècle. C'est une figure très controversée: pour ses détracteurs, elle est le plus bel exemple de charlatanisme et de mystification du siècle dernier, alors que pour ses admirateurs, c'est la plus grande magicienne que le monde ait connue. Madame Blavatsky est un merveilleux personnage de théâtre que dévore un feu intérieur et c'est une leçon de planète.

V. \& I. - Pour terminer, j'aimerais vous rappeler ce passage que vous écriviez dans votre premier livre, Comme une enfant de la terre: Les mots m'apparaissent comme des escaliers en spirale qui aboutissent à une porte. Qui s'ouvre. Sans revenir à toute l'importance de la métaphore de la porte et du seuil dans vos textes, qu'on a discutée un peu en abordant le sens du voyage et du passage dans ces mêmes écrits, pourriez-vous commenter sur quoi s'ouvre cette porte, pour vous, aujourd'hui?

J. Marchessault - Elle s'ouvre sur l'impulsion qui nous pousse les uns vers les autres. Sur la terre, c'est l'envolée la plus haute dont les 
humains sont capables. Et Jeanne, dans Des cailloux blancs pour les forêts obscures, ajoute: Je vois passer un ange, la tête rejetée en arrière, les ailes fermées. Il monte un escalier en spirale [...] Je gravis les marches derrière lui. Dans l'escalier, le lion de Bangor me précède, tournant les poignées, ouvrant les portes. J'essaie à mon tour. En vain. Les portes sont verrouillées. Cette lutte avec les portes et pas un bruit [...] Père, oh! mon Père, aie pitié de tes enfants. Ne laisse pas les portes se refermer à jamais. Un par un, une par une, individuellement, nous sommes indispensables. Regarde-nous! Nous ne sommes déjà plus des chairs. Nous ne serons jamais plus des bêtes sauvages. Nous voulons résider ailleurs, comme les oiseaux, comme les étoiles. 